\title{
MAPPINGS OF THE INTERVAL WITH FINITELY MANY PERIODIC POINTS HAVE ZERO ENTROPY
}

\begin{abstract}
LOUIS BLOCK ${ }^{1}$
AbStract. It is shown that if $f$ is a continuous map of a closed interval into itself, and $f$ has finitely many periodic points, then the topological entropy of $f$ is zero.
\end{abstract}

1. Introduction. Let $I$ denote a closed interval and $C^{0}(I, I)$ denote the space of continuous functions of $I$ into itself. For $f \in C^{0}(I, I)$ let ent $(f)$ denote the topological entropy of $f$ (see [1], [2], [4], or [6] for a definition). The main result of this paper is the following.

THEOREM A. Let $f \in C^{0}(I, I)$ and suppose that $f$ has finitely many periodic points. Then $\operatorname{ent}(f)=0$.

To prove Theorem A we prove the following theorem. Let $\Omega(f)$ denote the nonwandering set (see $\$ 2$ for definitions).

THEOREM B. Let $f \in C^{0}(I, I)$. Suppose $f$ has finitely many periodic points, and all periodic points of $f$ are fixed points of $f$. Then $\Omega(f)$ is the set of fixed points of $f$.

Theorem A follows easily from Theorem B, using the following two elementary facts (see $\$ 3$ for details).

Lemma 1. (proved by Adler, Konheim and McAndrew in [1]). Let $f$ be a continuous map of a compact topological space and let $n$ be a positive integer. Then $\operatorname{ent}\left(f^{n}\right)=n \cdot \operatorname{ent}(f)$.

LEMMA 2 (proved by Bowen [6]). Let $f$ be a continuous map of a compact metric space and suppose $\Omega(f)$ is finite. Then ent $(f)=0$.

We remark that this paper uses some results from [3] which will be stated in §2. The main result of [3], which is somewhat related to Theorem B, is the following.

Received by the editors January 31, 1977.

Key words and phrases. Topological entropy, periodic point, nonwandering set, unstable manifold.

AMS (MOS) subject classifications (1970). Primary 54H20.

${ }^{1}$ Partially supported by NSF grant MCS 76-05822. 
TheOrem C. Let $f \in C^{0}(I, I)$ and suppose $\Omega(f)$ is finite. Then $\Omega(f)$ is the set of periodic points of $f$.

We conclude this section by noting that Theorems B and C leave open the following question.

If $f \in C^{0}(I, I)$ and $f$ has finitely many periodic points, is $\Omega(f)$ the set of periodic points of $f$ ?

2. Preliminary definitions and results. Let $f \in C^{0}(I, I)$. Let $f^{0}$ denote the identity map. For any positive integer $n$, we define $f^{n}$ inductively by $f^{1}=f$ and $f^{n}=f \circ f^{n-1}$. A point $x \in I$ is said to be periodic if $f^{n}(x)=x$, for some positive integer $n$. A point $x \in I$ is said to be eventually periodic, if $f^{n}(x)$ is periodic for some positive integer $n$. We say $x \in I$ is wandering if for some neighborhood $W$ of $x, f^{n}(W) \cap W=\varnothing$ for every positive integer $n$. The set of points which are not wandering is called the nonwandering set and is denoted $\Omega(f)$.

Now, let $f \in C^{0}(I, I)$ and let $p$ be a fixed point of $f$. We define the unstable manifold $W^{u}(p, f)$ and one-sided unstable manifold $W^{u}(p, f,+)$ as follows. Let $x \in W^{u}(p, f)$ if for any neighborhood $V$ of $x, x \in f^{n}(V)$ for some positive integer $n$. Let $x \in W^{u}(p, f,+)$ if for any interval $K$ with left endpoint $p, x \in f^{n}(K)$ for some positive integer $n$. It is easy to verify that $W^{u}(p, f)$ and $W^{u}(p, f,+)$ are connected.

We will use the following lemma, which is proved in $\$ 2$ of [3]:

LEMMA 3. Let $f \in C^{0}(I, I)$ and let $p$ be a fixed point of $f$. Let $\overline{W^{u}(p, f)}$ denote the closure of $W^{u}(p, f)$. Then any element of $\overline{W^{u}(p, f)}-W^{u}(p, f)$ is periodic.

We will also use the following theorem from [3] (see Theorem 7 of $\$ 3$ of [3]):

THEOREM 4. Let $f \in C^{0}(I, I)$. Suppose $f$ has finitely many periodic points and $p$ is a fixed point of $f$. If $x \in W^{u}(p, f)$ and $f(x)=p$, then $x=p$.

Since $f\left(W^{u}(p, f)\right) \subset W^{u}(p, f)$ the following corollary follows immediately from Theorem 4.

Corollary 5. Let $f \in C^{0}(I, I)$. Suppose $f$ has finitely many periodic points, and $p$ is a fixed point of $f$. If $x \in W^{u}(p, f)$ and $f^{m}(x)=p$ for some positive integer $m$, then $x=p$.

We conclude this section by proving the following lemma.

LEMMA 6. Let $f \in C^{0}(I, I)$. Suppose that $f$ has finitely many periodic points, and all periodic points of $f$ are fixed points of $f$. Let $F$ denote the union of the set of fixed points of $f$ and the set of endpoints of $I$. Let $p$ and $q$ be adjacent elements of $F$ with $p<q$ (i.e.p $\in F, q \in F$, and $(p, q) \cap F=\varnothing)$. Suppose for some $y \in(p, q), f(y)<y$. Then for any $x \in(p, q)$ and any positive integer $n$, $f^{n}(x)<x$. 
Proof. Since $f(y)<y$ for some $y \in(p, q)$ and $(p, q)$ has no fixed points, $f(x)<x \forall x \in(p, q)$. Also, it follows that $f(p)=p$. This is true because if $f(p) \neq p$, then $p$ is the left endpoint of $I$ and $f(p)>p$. But $f(p)>p$ and $f(y)<y$ imply the existence of a fixed point in $(p, y)$, a contradiction.

Let $n$ be a positive integer. First, suppose $q$ is a fixed point of $f$. Since $f(p, q) \supset(p, q)$, there is a point $c \in(p, q)$ with $f^{r}(c) \in(p, q)$ for $r=$ $1, \ldots, n$. Hence $f^{n}(c)<c$. Since $f^{n}$ has no fixed points in $(p, q), f^{n}(x)<$ $x \forall x \in(p, q)$.

Now, suppose that $q$ is not a fixed point of $f$. Then $q$ is the right endpoint of $I$ and $f^{n}(q)<q$. Again, since $f^{n}$ has no fixed points in $(p, q), f^{n}(x)<$ $x \forall x \in(p, q)$. Q.E.D.

\section{Proofs of Theorems A and B.}

THEOREM B. Let $f \in C^{0}(I, I)$. Suppose $f$ has finitely many periodic points, and all periodic points of $f$ are fixed points of $f$. Then $\Omega(f)$ is the set of fixed points of $f$.

Proof. Let $y \in I$ be any point which is not fixed. We will show that $y$ is wandering.

Let $F$ denote the union of the set of fixed points of $f$ and the set of endpoints of $I$. There is a unique pair of elements of $F, p_{0}$ and $q_{0}$, such that $p_{0}<q_{0},\left(p_{0}, q_{0}\right) \cap F=\varnothing$, and $y \in\left[p_{0}, q_{0}\right]$. Since $y$ is not a fixed point of $f$, either $f(y)<y$ or $f(y)>y$. Without loss of generality, we may assume that $f(y)<y$. This implies, by Lemma 6 , that for any $x \in\left(p_{0}, q_{0}\right)$ and any positive integer $n, f^{n}(x)<x$. Hence $f\left(p_{0}\right)=p_{0}$. We separate the proof into two cases.

Case $1 . y$ is eventually periodic.

Then for some fixed point $e$ of $f$, and some positive integer $m, f^{m}(y)=e$. Note that by Lemma $6, e<y$. By Corollary $5, y \notin W^{u}(e, f)$. Hence, by Lemma $3, y \notin \overline{W^{u}(e, f)}$, since $y$ is not periodic. Thus, for some $a \in I$ with $e<a<y, a \notin W^{u}(e, f)$.

Since $a \notin W^{u}(e, f)$, there is an open interval $N$ containing $e$ such that $a \notin \cup_{n>0} f^{n}(N)$. Let $W=(b, c)$ be an open interval with $a<b<y<c$, and $f^{m}(W) \subset N$, and $f^{r}(W) \cap W=\varnothing$ for $r=1, \ldots, m$. Since $\cup_{n>0} f^{n}(N)$ is a connected set containing $e$ but not $a, W \cap\left(\cup_{n>0} f^{n}(N)\right)=\varnothing$. Hence $f^{r}(W) \cap W=\varnothing$ for every positive integer $r$. Thus, $y$ is wandering.

Case $2 . y$ is not eventually periodic.

First suppose that for some positive integer $n, f^{n+1}(y)<f^{n}(y)$. This implies that $f^{n}(y)$ is not the left endpoint of $I$. Since $f^{n}(y)<y, f^{n}(y)$ is not the right endpoint of $I$. Thus, $f^{n}(y) \notin F$. Hence, there is a unique pair of elements of $F, p_{n}$ and $q_{n}$, such that $p_{n}<q_{n},\left(p_{n}, q_{n}\right) \cap F=\varnothing$ and $f^{n}(y) \in$ $\left(p_{n}, q_{n}\right)$. By Lemma 5 , for every positive integer $m, f^{m}(x)<x \forall x \in\left(p_{n}, q_{n}\right)$.

Let $V$ be a neighborhood of $y$ such that $f^{r}(V) \cap V=\phi$ for $r=1, \ldots, n$ and $f^{n}(V) \subset\left(p_{n}, q_{n}\right)$. Then $f^{n}(V)$ is to the left of $V$, and for every positive 
integer $m, f^{m}(x)<x \forall x \in f^{n}(V)$. Hence $f^{r}(V) \cap V=\phi$ for every positive integer $r$. Thus, $y$ is wandering.

Now, suppose that $f^{n+1}(y) \geqslant f^{n}(y)$ for every positive integer $n$. Since $y$ is not eventually periodic, $f^{n+1}(y)>f^{n}(y)$ for every positive integer $n$. Let $z$ be the limit of the increasing sequence $\left(f^{n}(y)\right)$. Then $z>f^{n}(y)$ for every positive $n, z \leqslant p_{0}$ and $f(z)=z$.

We claim that $y \notin \overline{W^{u}(z, f,+)}$. Suppose that $y \in \overline{W^{u}(z, f,+)}$. Recall that for every positive integer $m, f^{m}(x)<x \forall x \in\left(p_{0}, q_{0}\right)$. Hence for any $x>p_{0}, x \notin \overline{W^{u}\left(p_{0}, f,+\right)}$. In particular, $y \notin \overline{W^{u}\left(p_{0}, f,+\right)}$. Hence $z \neq p_{0}$. Since $z \leqslant p_{0}$, this implies that $z<p_{0}$. Now since $\overline{W^{u}(z, f,+)}$ is connected, $[z, y] \subset \overline{W^{u}(z, f,+)}$. But $f(y)<z$ and $f\left(p_{0}\right)>z$. Hence, for some $x \in$ $\left(p_{0}, y\right), f(x)=z$. Since $x \in W^{u}(z, f,+)$, this is a contradiction, by Theorem 4. This establishes our claim that $y \notin \overline{W^{u}(z, f,+)}$.

Since $\left.y \notin \overline{W^{u}(z, f,+}\right)$, there is an interval $D$ with left endpoint $z$, such that $y \notin \overline{\bigcup_{n \geqslant 0} f^{n}(D)}$. Let $w$ be the greatest element of $\{x \in F: x<z\}$. Since $z$ is the limit of the increasing sequence $\left(f^{n}(y)\right), f(x)>x \forall x \in(w, z)$, by Lemma 6 . Hence there is an interval $K$ with right endpoint $z$, such that $f(K) \subset K \cup D$.

For some positive integer $j, f^{j}(y)$ is in the interior of $K$. Let $B$ be a neighborhood of $y$ with $B \cap \overline{\left(U_{n \geqslant 0} f^{n}(D)\right)}=\varnothing$ and $f^{r}(B) \cap B=\varnothing$ for $r=1, \ldots, j$, and $f^{j}(B) \subset K$. Then $f^{r}(B) \cap B=\varnothing$ for every positive integer $r$. Hence $y$ is wandering. Q.E.D.

THEOREM A. Let $f \in C^{0}(I, I)$ and suppose $f$ has finitely many periodic points. Then $\operatorname{ent}(f)=0$.

Proof. Let $n$ be the product of the periods of all periodic points of $f$. Then all periodic points of $f^{n}$ are fixed points of $f^{n}$. By Theorem $\mathrm{B}, \Omega\left(f^{n}\right)$ is the set of fixed points of $f^{n}$. In particular, $\Omega\left(f^{n}\right)$ is finite. Hence $\operatorname{ent}\left(f^{n}\right)=0$, by Lemma 2. Thus, by Lemma 1 , ent $(f)=0$. Q.E.D.

\section{REFERENCES}

1. R. Adler, A. Konheim and M. McAndrew, Topological entropy, Trans. Amer. Math. Soc. 114 (1965), 309-319.

2. L. Block, An example where topological entropy is continuous, Trans. Amer. Math. Soc. 231 (1977), 201-213.

3. Continuous maps of the interval with finite nonwandering set, Trans. Amer. Math. Soc. (to appear).

4. Topological entropy at an $\Omega$-explosion, Trans. Amer. Math. Soc. (to appear).

5. R. Bowen, Entropy for group endomorphisms and homogeneous spaces, Trans. Amer. Math. Soc. 153 (1971), 401-414.

6. Soc., Providence, R.I., 1970, pp. 23-41.

7. R. Bowen and J. Franks, The periodic points of maps of the disc and the interval, Topology 15 (1976), 337-442.

Department of Mathematics, University of Florida, Gainesville, Florida 32611 